\title{
Anti-diabetic agents and heart health: how to use new diabetes medications in a global strategy for the prevention of cardiovascular complications in type 2 diabetes
}

\author{
Carmine Gazzaruso ${ }^{1,2}$, Adriana Coppola ${ }^{1}$, Tiziana Montalcini ${ }^{3}$, Colomba Falcone ${ }^{2,4}$ \\ ${ }^{1}$ Diabetes and endocrine and metabolic diseases Unit and the Centre for Applied Clinical Research (Ce.R.C.A.) Clinical Institute "Beato Matteo" \\ (Hospital Group San Donato), Vigevano, Italy; ${ }^{2}$ Interdepartmental Center for Research in Molecular Medicine (CIRMC), University of Pavia, Pavia, \\ Italy; ${ }^{3}$ Department of Clinical and Experimental Medicine, Nutrition Unit, University Magna Grecia, Catanzaro, Italy; ${ }^{4}$ Department of Cardiology, \\ Istituti Clinici di Pavia e Vigevano, University Hospital, Pavia, Italy \\ Correspondence to: Carmine Gazzaruso, MD, PhD. Diabetes and endocrine and metabolic diseases Unit and the Centre for Applied Clinical Research \\ (Ce.R.C.A.) Clinical Institute "Beato Matteo”, Corso Pavia, 84, 27029 Vigevano, Italy. Email: c.gazzaruso@gmail.com. \\ Provenance: This is an invited Editorial commissioned by Section Editor Qi-Nan Wu, MD, PhD (Associate Professor of Endocrinology Department, \\ South West Hospital, Third Military Medical University, Diabetic Foot Diagnosis \& Treatment Medical Center of Chong Qing, Chong Qing, \\ China), and Guest Section Editor Xiaotian Lei [The First Hospital Affiliated to Army Medical University (Southwest Hospital), Chongqing, China]. \\ Comment on: Pagidipati NJ, Navar AM, Pieper KS, et al. Secondary Prevention of Cardiovascular Disease in Patients With Type 2 Diabetes Mellitus: \\ International Insights From the TECOS Trial (Trial Evaluating Cardiovascular Outcomes With Sitagliptin). Circulation 2017;136:1193-203.
}

Submitted Mar 06, 2018. Accepted for publication Mar 13, 2018.

doi: $10.21037 /$ atm.2018.03.30

View this article at: http://dx.doi.org/10.21037/atm.2018.03.30

Type 2 diabetes mellitus is a powerful cardiovascular (CV) risk factor (1). Diabetic patients have a 2 to 4 times increased $\mathrm{CV}$ risk and about $80 \%$ of them die from $\mathrm{CV}$ disease (CVD) (1-3). This elevated CV risk is in part due to hyperglycemia but is mainly caused by the features of the metabolic syndrome, such as insulin-resistance, lipid disorders, obesity, hypertension, hyperuricemia $(1,3)$. In fact, several studies showed that hyperglycemia may be a weak $\mathrm{CV}$ risk factor $(4,5)$ and that an intensive glycemic control may even increase CV mortality (4,5) if hypoglycemia occurs (6). In addition to CVD, heart failure (HF) represents an important cause of increased morbidity and mortality in people with diabetes, as it is more prevalent in these subjects than in the general population (1). HF in diabetes is caused both by coronary artery disease (CAD) and by a specific heart disease called diabetic cardiomyopathy (1). An increased CV morbidity and mortality in diabetes may be also due to the fact that vascular complications are often asymptomatic and thus untreated $(7,8)$. Therefore, new screening strategies and new predictors for asymptomatic CAD and peripheral artery disease (PAD) have been proposed (8-11). In addition, there may be an increased global CV morbidity and mortality due to diabetes, as diabetes is growing in pandemic proportions (2). Today it affects about 425 million subjects worldwide, but this number is projected to increase more than 628 million by 2045 (12). In other words, about $12 \%$ population will have diabetes.

\section{CV prevention in diabetes}

The reduction of $\mathrm{CV}$ morbidity and mortality in diabetes can be obtained not only by the treatment of hyperglycemia but also by the control of all associated $\mathrm{CV}$ risk factors. Therefore, diabetes drugs should ideally act with positive effects both on the glycemia and on the factors of the metabolic syndrome. In addition, it is important to use glucose-lowering medications with established CV safety. This need arose especially when in 2007 rosiglitazone was accused of increasing the risk for myocardial infarction and death (13).

\section{CV safety and efficacy of new diabetes medications}

After the rosiglitazone saga, Food and Drug Administration 
Table 1 CVOTs regarding new classes of glucose-lowering medications completed from 2008 until now

\begin{tabular}{|c|c|c|}
\hline Name of CVOT & Drug & Completed in \\
\hline TECOS & Sitagliptin & 2015 \\
\hline EXAMINE & Alogliptin & 2014 \\
\hline SAVOR-TIMI 53 & Saxagliptin & 2014 \\
\hline \multicolumn{3}{|c|}{ Glucagon-like peptide1 receptor agonists } \\
\hline EXSCEL & Exenatide LAR & 2017 \\
\hline SUSTAIN-6 & Semaglutide & 2016 \\
\hline LEADER & Liraglutide & 2015 \\
\hline ELIXA & Lixisenatide & 2015 \\
\hline \multicolumn{3}{|c|}{ Sodium-glucose cotransporter 2 inhibitors } \\
\hline EMPA-REG OUTCOME & Empagliflozin & 2015 \\
\hline \multicolumn{3}{|c|}{$\begin{array}{l}\text { TECOS, Trial Evaluating Cardiovascular Outcomes with Sitagliptin; EXAMINE, Examination of Cardiovascular Outcomes with Alogliptin } \\
\text { versus Standard of Care; SAVOR-TIMI 53, Saxagliptin Assessment of Vascular Outcomes Recorded in Patients with Diabetes Mellitus- } \\
\text { Thrombolysis in Myocardial Infarction 53; EXSCEL, Exenatide Study of Cardiovascular Event Lowering; SUSTAIN-6, Trial to Evaluate } \\
\text { Cardiovascular and Other Long-term Outcomes with Semaglutide in Subjects with Type } 2 \text { Diabetes; LEADER, Liraglutide Effect and Action } \\
\text { in Diabetes: Evaluation of Cardiovascular Outcome Results; ELIXA, Evaluation of Lixisenatide in Acute Coronary Syndrome; CANVAS, } \\
\text { Canagliflozin Cardiovascular Assessment Study. EMPA-REG OUTCOME, Empagliflozin, Cardiovascular Outcomes, and Mortality in Type } 2 \\
\text { Diabetes; COVT, cardiovascular outcomes trial; LAR, long-acting release. }\end{array}$} \\
\hline
\end{tabular}

(FDA) and European Medicine Agency (EMA) required new glucose-lowering medications to demonstrate CV safety, resulting in specific clinical trials, called CV Outcomes Trials (CVOTs). The primary composite outcome of the CVOTs on new diabetes medications comprised similar elements, namely the following major adverse cardiovascular events (MACE): CV death, nonfatal myocardial infarction and nonfatal stroke. There may be differences in secondary endpoints among the CVOTs. CVTOs regarded three new classes of diabetes drugs: dipeptidyl peptidase IV (DPP-IV) inhibitors or gliptins, Glucagon-like peptide-1 (GLP-1) receptors agonists (GLP-1 Ras) or incretin-mimetics, and sodium-glucose cotransporter-2 (SGLT-2) inhibitors. Since issuing this new guidance, a lot of major CVOTs have been completed until now and for some of them the results have been positively surprising. Table 1 summarizes all completed CVOTs regarding new glucose-lowering medications.

These trials have unexpectedly shown that some of these new medications can give $\mathrm{CV}$ benefits independently of their action on glycemic control. In addition, they have shown favorable effects on several $\mathrm{CV}$ risk factors associated to diabetes.
A statistically significant reduction in $\mathrm{CV}$ risk was first observed in the Empagliflozin, CV Outcomes, and Mortality in Type 2 Diabetes (EMPA-REG OUTCOME) trial that randomized more than 7,000 diabetic patients with vascular complications to receive the SGLT-2 inhibitor empagliflozin or placebo. After a 3.1-year period, patients receiving empagliflozin had a $14 \%$ reduction in the primary composite outcome, a $38 \%$ reduction in $\mathrm{CV}$ death, and a $35 \%$ reduction in HF hospitalizations (14). Interestingly, empagliflozin showed a reduction in weight, waist circumference, systolic and diastolic blood pressure, and acid uric levels with respect to placebo. To understand whether positive effects on $\mathrm{CV}$ risk and on risk factors other than glycemia may be due to the whole class of SGLT2 inhibitors data from trials and real-world studies are accumulating.

The Canagliflozin Cardiovascular Assessment Study (CANVAS) program comprised two sister trials (CANVAS and CANVAS-R) that was designed to evaluate CV safety and efficacy of canagliflozin. The authors included more than 10,000 diabetic people with vascular complications or at high CV risk. Patients taking canagliflozin had a $14 \%$ 
reduction in the primary composite outcome and a $33 \%$ reduction in HF hospitalizations (15). However, in this trial a statistically significant doubling incidence of amputations was documented in the canagliflozin group; this higher incidence of amputations may be due to a personal history of amputation or PAD at baseline.

Interestingly, a large real-world study compared three approved SGLT-2 inhibitors (empagliflozin, canagliflozin and dapagliflozin) versus other diabetes drugs in over 300,000 patients from six countries. In diabetic patients free of CVD at baseline SGLT-2 inhibitors were able to lower the risk of death by $51 \%$ and of $\mathrm{HF}$ hospitalizations by $39 \%$ (16).

Taken together, all these data clearly suggest that CV protection due to SGLT-2 inhibitors is independent of glucose-lowering and is not fully explained by the reduction in associated $\mathrm{CV}$ risk factors. Therefore, additional mechanisms have been hypothesized (17).

After the EMPA-REG OUTCOME trial, another CVOT showing CV benefits due to a new diabetes medication was published: the Liraglutide Effect and Action in Diabetes: Evaluation of Cardiovascular Outcome Results (LEADER) trial (18). It investigated liraglutide that belongs to GLP-1 RAs. More than 9,340 diabetic patients at high CV risk were randomized to liraglutide or placebo. After a 3.8-year period, patients under liraglutide had a $13 \%$ reduction in the primary composite outcome: this results was especially due to reduction in $\mathrm{CV}$ death. Then, another GLP-1 RA, semaglutide, showed important CV benefits (19). The Trial to Evaluate Cardiovascular and Other Long-term Outcomes with Semaglutide in Subjects with Type 2 Diabetes (SUSTAIN-6) trial randomized more than 3,200 diabetic patients with vascular complications, chronic kidney disease or high $\mathrm{CV}$ risk due to the presence of additional $\mathrm{CV}$ risk factors to semaglutide or placebo. Patients treated with semaglutide had a $26 \%$ reduction in the primary outcome, mostly due to the reduction in nonfatal stroke.

The Evaluation of Lixisenatide in Acute Coronary Syndrome (ELIXA) trial explored CV safety of lixisenatide, another GLP-1 RA, and was published before the LEADER study (20). It confirmed CV safety of lixisenatide but did not show any reduction in $\mathrm{CV}$ events. In the recent Exenatide Study of Cardiovascular Event Lowering (EXSCEL) trial, the once-weekly GLP-1 RA exenatide showed CV safety, but it was not superior with respect to efficacy, even if the $\mathrm{P}$ value was very near to the statistical significance $(0.06)(21)$.

As it is possible to see, CVOTs regarding the class of GLP-1 RAs did not give homogeneous results on CV benefits, but this may be due to differences in the features of people enrolled into the studies. However, it is important to remember that all GLP-1 RAs are associated to weight loss and decrease in blood pressure. A slight increase in heart rate and neutral effects on HF hospitalizations were also documented.

Another new class of glucose-lowering medications tested for CV safety is that of DPP-IV inhibitors. At the moment results from four large CVOTs are available in the literature. In these trials saxagliptin, alogliptin, sitagliptin and omarigliptin showed CV safety, even if a decrease in MACE was not observed (22-25). Nevertheless, two of these trials (Saxagliptin Assessment of Vascular Outcomes Recorded in Patients with Diabetes MellitusThrombolysis in Myocardial Infarction 53 -SAVOR-TIMI 53- and Examination of CV Outcomes with Alogliptin versus Standard of Care-EXAMINE-) suggested that saxagliptin and alogliptin may give a higher risk for HF hospitalizations. In particular SAVOR TIMI 53 showed that patients treated with saxagliptin had $27 \%$ higher risk for HF hospitalizations compared to placebo (22). In the EXAMINE trial a higher incidence of HF hospitalizations was not observed in the group of patients taking alogliptin than in placebo group (23). However, among the subjects without a history of $\mathrm{HF}$ at baseline the risk for $\mathrm{HF}$ hospitalizations was $76 \%$ greater in people treated with alogliptin than controls. Reassuring data came from the Trial Evaluating Cardiovascular Outcomes with Sitagliptin (TECOS) trial: indeed patients treated with sitagliptin did not have an increased risk for HF hospitalization (24) and this was confirmed by further analyses (26). The recent trial with omarigliptin showed that no increase in HF hospitalization was observed in subjects talking DPP-IV inhibitors when compared to controls (25).

To better understand the potential risk for $\mathrm{HF}$ hospitalization due to DPP-IV inhibitors meta-analyses and retrospective analyses on large databases were exploited. A meta-analysis found that a slight increase in the risk for HF hospitalization in people treated with gliptins (27), but another one with 54 studies did not (28). However, in the latter meta-analysis an increased risk for $\mathrm{HF}$ was seen in subjects taking saxagliptin, but not in people treated with other gliptins (28). The Canadian Network for Observational Drug Effect studies retrospectively examined administrative health electronic data of $1,499,650$ patients with 29,741 HF hospitalizations. The comparison between gliptins and other diabetes medications did not show any increased risk for HF hospitalization in diabetic patients 
with or without a history of HF (29). Similar results were obtained by an analysis of a large database made by the FDA (30). The lack of an increased HF risk was observed also in people treated with saxagliptin and alogliptin (30).

On the basis of these data, we can affirm that CV safety of DPP-IV inhibitors in term of MACE occurrence is certain. On the contrary the effect of gliptins on HF risk remains to be definitively elucidated. Data available in the literature suggest that there may be a higher risk for $\mathrm{HF}$, but that this risk is not a class effect. However, FDA recommended discontinuation of saxagliptin or alogliptin if HF occurs, and label for sitagliptin and linagliptin was modified to include a warning that other gliptins may increase the risk for HF hospitalization.

\section{Prevention of CV complications in clinical practice: a global approach}

Prevention of $\mathrm{CV}$ complications and death in diabetes remains a big problem for the clinicians. Indeed, as recently reviewed by Giugliano, in type 2 diabetes more intensive glycemic control leads to a slight reduction in MACE (about $9 \%)(4,5)$. This implies that the vascular residual risk is $91 \%$. Therefore, appropriate strategies should be adopted in daily clinical practice to adequately reduce $\mathrm{CV}$ risk. It is well-known that only a global intensive control of all CV risk factors can give a significant reduction of $\mathrm{CV}$ events in diabetes (31). Even if this evidence is well-known from many years, a large percentage of diabetic patients do not achieve the goals for $\mathrm{CV}$ risk factors both in primary and secondary prevention of CVD $(32,33)$.

A recent large study has evaluated the degree to which secondary prevention goals are met globally in diabetic patients with overt CVD (34). Five parameters have been taken into account: aspirin use, lipid control, blood pressure control, ACE-inhibitors or ARBs use, and non-smoking status. The authors used data from the TECOS trial. They found that among 13,616 diabetic patients and known CVD from 38 countries only $29 \%$ of them achieved all 5 secondary prevention parameters at baseline, even if $71.8 \%$ achieved at least 4 parameters. There were differences in the degree to which secondary prevention goals were met among world regions and countries. Only $58 \%$ of people attained blood pressure control and this was the lowest overall attainment, while non-smoking status was the highest (89\%). According to the authors these results have some important clinical implications. Indeed, this study has well documented that diabetic patients with CVD are still being undertreated globally with respect to secondary prevention, in particular with respect to blood pressure control. Therefore, these gaps in care, if filled, can provide important opportunities to greatly improve $\mathrm{CV}$ prognosis in very high $\mathrm{CV}$ risk patients (34).

To further reduce the risk for $\mathrm{CV}$ events in diabetic patients, especially in those with proven CVD, SGLT2 inhibitors and GLP-1 RAs should be used. Both can reduce associated $\mathrm{CV}$ risk factors, in particular obesity and hypertension, and the global CV risk independently of other risk factors. In addition, SGLT-2 may be an ideal choice in patients with $\mathrm{HF}$.

When patients have a glycemic control not far from the target DPP-IV inhibitors may represent a good choice, especially if the physician believes that weight excess can be treated with lifestyle modifications alone. This class should be avoided in patients with HF and saxagliptin and alogliptin should be avoided also in patients at risk for HF.

All these new therapeutic options should be always added to metformin that remains the first-line treatment, according to the current guidelines (35). On the other hand, several studies have documented CV safety of metformin and its positive effects on lipid profile, body weight, endothelial function, oxidative stress (36). In addition, metformin is safe and is able to give $\mathrm{CV}$ benefits, even in subjects with HF and in older patients (37-39).

It is important to remember that new glucose-lowering medications and metformin share the same characteristic of having a very low risk for hypoglycemia. Indeed, as already reported, hypoglycemia represents a strong $\mathrm{CV}$ risk factor (6). In addition, the occurrence of hypoglycemia may explain some neutral or negative results on $\mathrm{CV}$ outcomes when intensive glycemic control was obtained with drugs associated to high risk for hypoglycemia, such as sulfonylureas or insulin $(4,5,36)$.

Another important tool to decrease CV morbidity and mortality is to deliver to all the patients a structured patient therapeutic education, as recently suggested by several data $(3,40,41)$. Ongoing trials should elucidate the most effective methods by which patient education can give the best results in terms of $\mathrm{CV}$ prevention (42).

At last, it is important outline that $10-30 \%$ of diabetic patients may have an asymptomatic CAD (43). Some large randomized studies did not support the idea that a systematic screening for silent CAD may give CV benefits $(44,45)$, even if some studies documented an improvement in $\mathrm{CV}$ prognosis $(7,46)$. Therefore, current European Society Cardiology guidelines suggest to better define the real 
Table 2 A global strategy for cardiovascular prevention in type 2 diabetes

Obtain an intensive glycemic control

Achieve an intensive control of all associated cardiovascular risk factors

When possible, prefer diabetes medications at low risk for hypoglycemia

When tolerated, use metformin as the first-line treatment

Use SGLT2-inhibitors or GLP-1 receptor agonists in patients with known vascular complications or at high risk for vascular complications

Avoid saxagliptin and alogliptin in patients with heart failure or at risk for heart failure

Prefer SGLT2 inhibitors in patients with heart failure

Deliver to all the patients a structured patient therapeutic education

Screen for asymptomatic coronary artery disease and peripheral artery disease

impact that screening for silent CAD may have on the prognosis of the patients (47). Indeed, in patients with silent $\mathrm{CAD}$ a more strict control of $\mathrm{CV}$ risk factors may be requested on the basis of a secondary prevention strategy, an anti-ischemic therapy may be started and a revascularization may be performed, when appropriate. All these actions may improve the prognosis (7). The screening for asymptomatic PAD may be another important tool to identify and early treat diabetic patients at high $\mathrm{CV}$ risk (8).

Table 2 summarizes all the possible preventive measures to minimize the $\mathrm{CV}$ risk in people with type 2 diabetes.

\section{Conclusions}

Many recent findings have thrown a new light on how to greatly reduce the residual vascular risk in type 2 diabetic patients. It is necessary to obtain a strict glycemic control by using drugs with a low risk for hypoglycemia. In addition, the patients should achieve the goals for all associated CV risk factors and receive a structured patient therapeutic education. Screening for asymptomatic CAD and PAD may help to adopt a secondary prevention strategy and to treat them with appropriate therapies. At last, newer glucoselowering medications can be very helpful in the treatment of diabetes because of their CV safety, action on associated $\mathrm{CV}$ risk factors and very low risk for hypoglycemia. Some of them can even give additional CV benefits that are independent of all the above preventive measures to reduce CV morbidity and mortality.

\section{Acknowledgements}

None.

\section{Footnote}

Conflicts of Interest: The authors have no conflicts of interest to declare.

\section{References}

1. Low Wang CC, Hess CN, Hiatt WR, et al. Clinical Update: Cardiovascular Disease in Diabetes Mellitus: Atherosclerotic Cardiovascular Disease and Heart Failure in Type 2 Diabetes Mellitus - Mechanisms, Management, and Clinical Considerations. Circulation 2016;133:2459-502.

2. Sarwar N, Gao P, Seshasai SR, et al. Diabetes mellitus, fasting blood glucose concentration, and risk of vascular disease: a collaborative meta-analysis of 102 prospective studies. Lancet 2010;375:2215-22.

3. Coppola A, Sasso L, Bagnasco A, et al. The role of patient education in the prevention and management of type 2 diabetes: an overview. Endocrine 2016;53:18-27.

4. Giugliano D, Maiorino MI, Bellastella G, et al. Glycemic control in type 2 diabetes: from medication nonadherence to residual vascular risk. Endocrine 2018. [Epub ahead of print].

5. Giugliano D, Maiorino MI, Bellastella G, et al. Type 2 diabetes and cardiovascular prevention: the dogmas disputed. Endocrine 2018;60:224-8.

6. Lee AK, Warren B, Lee CJ, et al. The Association of Severe Hypoglycemia With Incident Cardiovascular Events and Mortality in Adults With Type 2 Diabetes. Diabetes Care 2018;41:104-11.

7. Gazzaruso C, Coppola A, Montalcini T, et al. Screening for asymptomatic coronary artery disease can reduce cardiovascular mortality and morbidity in type 2 diabetic 
patients. Intern Emerg Med 2012;7:257-66.

8. Gazzaruso C, Coppola A, Falcone C, et al. Transcutaneous oxygen tension as a potential predictor of cardiovascular events in type 2 diabetes: comparison with ankle-brachial index. Diabetes Care 2013;36:1720-5.

9. Gazzaruso C, Coppola A, Montalcini T, et al. Erectile dysfunction can improve the effectiveness of the current guidelines for the screening for asymptomatic coronary artery disease in diabetes. Endocrine 2011;40:273-9.

10. Gazzaruso C, Pujia A, Solerte SB, et al. Erectile dysfunction and angiographic extent of coronary artery disease in type II diabetic patients. Int J Impot Res 2006;18:311-5.

11. Gazzaruso C, Giordanetti S, De Amici E, et al. Relationship between erectile dysfunction and silent myocardial ischemia in apparently uncomplicated type 2 diabetic patients. Circulation 2004;110:22-6.

12. International Diabetes Federation. Available online: https://www.idf.org/e-library/epidemiology-research/ diabetes-atlas.html. Accessed 25 February 2018.

13. Nissen SE, Wolski K. Effect of rosiglitazone on the risk of myocardial infarction and death from cardiovascular causes. N Engl J Med 2007;356:2457-71.

14. Zinman B, Wanner C, Lachin JM, et al. Empagliflozin, Cardiovascular Outcomes, and Mortality in Type 2 Diabetes. N Engl J Med 2015;373:2117-28.

15. Neal B, Perkovic V, Mahaffey KW, et al. Canagliflozin and Cardiovascular and Renal Events in Type 2 Diabetes. N Engl J Med 2017;377:644-57.

16. Kosiborod M, Cavender MA, Fu AZ, et al. Lower Risk of Heart Failure and Death in Patients Initiated on SodiumGlucose Cotransporter-2 Inhibitors Versus Other GlucoseLowering Drugs: The CVD-REAL Study (Comparative Effectiveness of Cardiovascular Outcomes in New Users of Sodium-Glucose Cotransporter-2 Inhibitors). Circulation 2017;136:249-59.

17. Monica Reddy RP, Inzucchi SE. SGLT2 inhibitors in the management of type 2 diabetes. Endocrine 2016;53:364-72.

18. Marso SP, Daniels GH, Brown-Frandsen K, et al. Liraglutide and Cardiovascular Outcomes in Type 2 Diabetes. N Engl J Med 2016; 375:311-22.

19. Marso SP, Bain SC, Consoli A, et al. Semaglutide and Cardiovascular Outcomes in Patients with Type 2 Diabetes. N Engl J Med 2016;375:1834-44.

20. Pfeffer MA, Claggett B, Diaz R, et al. Lixisenatide in Patients with Type 2 Diabetes and Acute Coronary Syndrome. N Engl J Med 2015;373:2247-57.

21. Holman RR, Bethel MA, Mentz RJ, et al. Effects of Once-
Weekly Exenatide on Cardiovascular Outcomes in Type 2 Diabetes. N Engl J Med 2017;377:1228-39.

22. Scirica BM, Bhatt DL, Braunwald E, et al. Saxagliptin and cardiovascular outcomes in patients with type 2 diabetes mellitus. N Engl J Med 2013;369:1317-26.

23. White WB, Cannon CP, Heller SR, et al. Alogliptin after acute coronary syndrome in patients with type 2 diabetes. N Engl J Med 2013;369:1327-35.

24. Green JB, Bethel MA, Armstrong PW, et al. Effect of Sitagliptin on Cardiovascular Outcomes in Type 2 Diabetes. N Engl J Med 2015;373:232-42.

25. Gantz I, Chen M, Suryawanshi S, et al. A randomized, placebo-controlled study of the cardiovascular safety of the once-weekly DPP-4 inhibitor omarigliptin in patients with type 2 diabetes mellitus. Cardiovasc Diabetol 2017;16:112.

26. McGuire DK, Van de Werf F, Armstrong PW, et al. Association Between Sitagliptin Use and Heart Failure Hospitalization and Related Outcomes in Type 2 Diabetes Mellitus: Secondary Analysis of a Randomized Clinical Trial. JAMA Cardiol 2016;1:126-35.

27. Li L, Li S, Deng K, et al. Dipeptidyl peptidase-4 inhibitors and risk of heart failure in type 2 diabetes: systematic review and meta-analysis of randomised and observational studies. BMJ 2016;352:i610.

28. Kongwatcharapong J, Dilokthornsakul P, Nathisuwan S, et al. Effect of dipeptidyl peptidase-4 inhibitors on heart failure: A meta-analysis of randomized clinical trials. Int J Cardiol 2016;211:88-95.

29. Filion KB, Azoulay L, Platt RW, et al. A Multicenter Observational Study of Incretin-based Drugs and Heart Failure. N Engl J Med 2016;374:1145-54.

30. Toh S, Hampp C, Reichman ME, et al. Risk for Hospitalized Heart Failure Among New Users of Saxagliptin, Sitagliptin, and Other Antihyperglycemic Drugs: A Retrospective Cohort Study. Ann Intern Med 2016;164:705-14.

31. Gaede P, Pedersen O. Intensive integrated therapy of type 2 diabetes: implications for long-term prognosis. Diabetes 2004;53:S39-47.

32. Vaccaro O, Boemi M, Cavalot F, et al. The clinical reality of guidelines for primary prevention of cardiovascular disease in type 2 diabetes in Italy. Atherosclerosis 2008;198:396-402.

33. Kotseva K; EUROASPIRE Investigators. The EUROASPIRE surveys: lessons learned in cardiovascular disease prevention. Cardiovasc Diagn Ther 2017;7:633-9.

34. Pagidipati NJ, Navar AM, Pieper KS, et al. Secondary Prevention of Cardiovascular Disease in Patients With 
Type 2 Diabetes Mellitus: International Insights From the TECOS Trial (Trial Evaluating Cardiovascular Outcomes With Sitagliptin). Circulation 2017;136:1193-203.

35. American Diabetes Association. 8. Pharmacologic Approaches to Glycemic Treatment: Standards of Medical Care in Diabetes-2018. Diabetes Care 2018;41:S73-85.

36. Yandrapalli S, Jolly G, Horblitt A, et al. Cardiovascular benefits and safety of non-insulin medications used in the treatment of type 2 diabetes mellitus. Postgrad Med 2017;129:811-21.

37. Bossoni S, Mazziotti G, Gazzaruso C, et al. Relationship between instrumental activities of daily living and blood glucose control in elderly subjects with type 2 diabetes. Age Ageing 2008;37:222-5.

38. Mazziotti G, Bossoni S, Orini S, et al. Treatment with metformin is protective against limitations in instrumental activities of daily living in older subjects with type 2 diabetes mellitus. J Am Geriatr Soc 2009; 57:562-4.

39. Gazzaruso C, Coppola A, Luppi C, et al. Effect of different diabetes mellitus treatments on functional decline and death in elderly adults with diabetes mellitus. J Am Geriatr Soc 2013;61:666-7.

40. He X, Li J, Wang B, et al. Diabetes self-management education reduces risk of all-cause mortality in type 2 diabetes patients: a systematic review and meta-analysis. Endocrine 2017;55:712-31.

41. Gazzaruso C, Fodaro M, Coppola A. Structured therapeutic education in diabetes: is it time to re-write the chapter on the prevention of diabetic complications? Endocrine 2016;53:347-9.

Cite this article as: Gazzaruso C, Coppola A, Montalcini T, Falcone C. Anti-diabetic agents and heart health: how to use new diabetes medications in a global strategy for the prevention of cardiovascular complications in type 2 diabetes. Ann Transl Med 2018;6(10):195. doi: 10.21037/atm.2018.03.30
42. Coppola A, Luzi L, Montalcini T, et al. Role of structured individual patient education in the prevention of vascular complications in newly diagnosed type 2 diabetes: the INdividual Therapeutic Education in Newly Diagnosed type 2 diabetes (INTEND) randomized controlled trial. Endocrine 2018;60:46-9.

43. Gazzaruso C, Coppola A, Giustina A. Erectile dysfunction and coronary artery disease in patients with diabetes. Curr Diabetes Rev 2011;7:143-7.

44. Young LH, Wackers FJ, Chyun DA, et al. Cardiac outcomes after screening for asymptomatic coronary artery disease in patients with type 2 diabetes: the DIAD study: a randomized controlled trial. JAMA 2009;301:1547-55.

45. Muhlestein JB, Lappé DL, Lima JA, et al. Effect of screening for coronary artery disease using CT angiography on mortality and cardiac events in highrisk patients with diabetes: the FACTOR-64 randomized clinical trial. JAMA 2014;312:2234-43.

46. Faglia E, Mantero M, Quarantiello A, et al. Risk reduction of cardiac events by screening of unknown asymptomatic coronary artery disease in subjects with type 2 diabetes mellitus at high cardiovascular risk: an open-label randomized pilot study. Am Heart J 2005;149:e1-6.

47. Rydén L, Grant PJ, Anker SD, et al. ESC Guidelines on diabetes, pre-diabetes, and cardiovascular diseases developed in collaboration with the EASD: the Task Force on diabetes, pre-diabetes, and cardiovascular diseases of the European Society of Cardiology (ESC) and developed in collaboration with the European Association for the Study of Diabetes (EASD). Eur Heart J 2013;34:3035-87. 